DOI: $10.2478 / \mathrm{v} \cdot 10169-012-0010-3$

\title{
NUMERICAL IMPLEMENTATION OF MESHLESS METHODS FOR BEAM PROBLEMS
}

\author{
V.E. ROSCA ${ }^{1}$, V.M.A. LEITÃO ${ }^{2}$
}

\begin{abstract}
For solving a partial different equation by a numerical method, a possible alternative may be either to use a mesh method or a meshless method. A flexible computational procedure for solving 1D linear elastic beam problems is presented that currently uses two forms of approximation function (moving least squares and kernel approximation functions) and two types of formulations, namely the weak form and collocation technique, respectively, to reproduce Element Free Galerkin (EFG) and Smooth Particle Hydrodynamics (SPH) meshless methods. The numerical implementation for beam problems of these two formulations is discussed and numerical tests are presented to illustrate the difference between the formulations.
\end{abstract}

Key words: numerical methods; meshless formulation, EFG, SPH, beam discretisation.

\section{INTRODUCTION}

In engineering and science, one often has a number of data points, as obtained by sampling or some experiment, and tries to construct a function, which closely fits those data points. The so-called meshless methods construct approximations from a set of nodal data without the need for any (finite - element) a priori connectivity information between the nodes. In general, it is recommendable to preserve the local character of the numerical implementation. Meshless method allows to use local interpolation or approximation to represent the trial function with the values (or the fictitious values) of the unknown variable at some randomly located nodes.

There are two ways to construct approximations of a function using meshless methods:

- a continuous form, or reproducing kernel (RK) approximation.

A reproducing kernel $K$ is a function $K(\mathrm{x}, \mathrm{y}) \rightarrow \mathrm{R}$, such that all evaluation functions are continuous, and the functions can be reproduced by the kernel:

$$
\forall f, \quad\langle f(y), K(x, y)\rangle=f(x)
$$

\footnotetext{
${ }^{1}$ Lecturer, "Gheorghe Asachi” Technical University from Iasi, Faculty Of Civil Engineering and Building Services 43, Dimitrie Mangeron Blvd., 700050 Iasi, Romania, e-mail: roscave@ yahoo.com

2 Associate Professor, Technical University of Lisbon, Instituto Superior Técnico, 1 Rovisco Pais Street, 1049-001 Lisbon, Portugal. e-mail: vitor@civil.ist.utl.pt
} 
Accordingly, a function $u(\mathbf{x})$ can be approximated with $u^{h}(\mathbf{x})$ in a continuous way, by the integral transformation:

$$
u(x) \approx u^{h}(x)=\int_{\Omega_{y}} K(x, y) u(y) d \Omega_{y}
$$

Smoothed Particle Hydrodynamics (SPH), Lucy [1] and Reproducing Kernel Particle Method (RKPM), Liu, JUN and ZHANG [2] are two representative methods of RK.

- discrete form

There are several types of discrete approximation functions. Among these are: Moving least square (MLS) functions, Partition of unity (PU) functions, or hp-cloud functions, as representatives. Surveys can be found in (Li and LiU [3]).

The domain is discretized by a set of $N$ nodes, and the solution, $u(\mathbf{x})$, is approximated by a linear combination of nodal coefficients, $\hat{\mathrm{u}}_{I j}$, and of approximation functions, $h_{I j}(\mathbf{x})$,

$$
u(x) \approx u^{h}(x)=\sum_{I=1}^{N} \sum_{j=1}^{m} h_{I j}(x) \hat{u}_{I j}
$$

where $m$ denotes the number of coefficients per node.

In addition to these requirements, one often forces the approximation functions to satisfy other properties. For example, if the approximation functions satisfy the interpolation property (functions associated with a node are unity at that node and zero at all other nodes) and $m=1$ (see Eq.(1.3)), then nodal coefficients can be interpreted as the solution evaluated at the node. In this case, we refer to the approximation function as an interpolation function.

The approximations functions constructed in continuous or in discrete way are used as approximations of the strong forms of partial differential equations (PDEs), and those serving as approximations of the weak forms of PDEs to set up a linear system of equations.

To approximate the strong form of a PDE, the partial differential equation is usually discretized by specific collocation technique. The SPH is a representative method for the strong form collocation approach.

To approximate the weak form of a PDE various Galerkin weak formulations are used. Another imposed attribute arises from the condition that the resulting matrix system equation obtained by strong or weak form to be banded. This is possible when the approximation functions exhibit local support. In this case, each node has a domain of influence, a region of the domain in which the approximation functions for that node are non-zero. A large domain of influence will result in a node being influenced by nodes far away, while a small domain of influence will result in a node being influenced only by close neighbors. If the support is small, there is less coupling between nodes, and the system matrix has a small bandwidth. 
In this paper, we will describe how the meshless approximation is constructed for two representative families: interpolations based on kernel methods and interpolations based on Moving Least Squares (MLS) functions.

Kernel methods are briefly summarized in section 2; MLS approximation functions are derived from a generalization of a weighted least square fit and are described in section 3.

Some concepts weak formulations are recalled in section 4, with special attention on the Galerkin formulation and, in particular, on the Element-Free Galerkin (EFG) method.

Numerical comparison between these two formulations is performed in section 5 . Numerical applications for beam problems are obtained by implementing various quadrature techniques to perform the integrations of the system equations in EFG method or for discretize the continuous form of the displacement of the SPH.

\section{Smooth Particle Hydrodynamics (SPH)}

The kernel estimate of a function is the integral transformation of a function $u(\mathrm{x})$ to $u^{h}(\mathrm{x})$ :

$$
u(x) \approx u^{h}(x)=\int_{\Omega_{y}} w(x-y) u(\mathrm{y}) d \Omega_{y}
$$

where $u^{h}(\mathrm{x})$ is the 'reproduced' function of $u(\mathrm{x})$, and Eq. (2.1) is called the reproducing equation and $w(\mathrm{x}-\mathrm{y})$ is known as the kernel (or weight) of the transformation.

If the kernel (or weight function) is equal to the Dirac function $\delta(\mathrm{x}-\mathrm{y})$, then $u^{h}(\mathrm{x})$ $\rightarrow u(\mathbf{x})$. In SPH, the kernel is selected to have a small compact support, such that $u^{h}(\mathbf{x})$ approximates $u(\mathbf{x})$. Note that the Eq. (2.1) is a continuous form of an approximation. Usually a positive function, such as the Gaussian function or spline functions are usually employed.

For the purposes of developing approximation, discrete analogous of Eq. (2.1) are needed. The discrete form of Eq. (2.1) is obtained by numerical quadrature of the right-hand side.

For an arbitrary PDE given by Eq. (2.1), using SPH approximation leads to:

$$
u(x) \approx u^{h}(x)=\int_{\Omega_{y}} w(x-y) u(\mathrm{y}) d \Omega_{y}=\sum_{I=1}^{N} w_{I}(x) \Delta V_{I} u\left(x_{I}\right)
$$

Commonly used weight functions are exponential, the cubic spline and the quartic spline. Once the quadrature is performed, the approximation can then be rewritten in the readily recognized form:

$$
u^{h}(x)=\sum_{I}^{N} \phi_{I}(x) \cdot \hat{u}_{I}
$$


where

$$
\phi_{I}(x)=\sum_{I}^{N} w_{I}(x) \cdot \Delta V_{I}
$$

From Eq. (2.4), because $\Delta \mathrm{V}_{I}$ stands for integration weights, consistency cannot be reached at boundaries, where

$$
\sum_{I=1}^{N} w_{I}(x) \Delta V_{I} \neq 1
$$

The lack of consistency near boundaries leads to a solution deterioration near the domain boundaries.

In spite of absence of linear consistency, SPH methods have provided good solutions to second-order partial differential equations.

Improvements of the standard SPH method are still an active research area. There exist a number of proposed ideas for correction of SPH addressing issues such as instability, boundary conditions and consistency. An overview and further references can be found in (Li and Liu [3], (Belytschko et. al [4]), and Randles and Libersky $[5])$.

\section{Construction of MLS interpolants}

As in the corrected SPH methods commented in the previous section, the interpolations based on a Moving Least Squares (MLS) development can be considered as an improvement of the SPH method. However, the MLS interpolations are usually used to discretize a Galerkin formulation, and thus, accuracy and consistency in both the interpolation and its derivatives are needed in the entire domain.

Given a set of scattered nodes in $(d=1 \ldots 3)$ with prescribed nodal data, a surface approximation can be constructed without the need for any (finite - element) a priori connectivity information between the nodes. This viewpoint is adopted in meshless Galerkin methods, where well-known methods from data approximation theory (Lancaster and Salkauskas [6]) are used to construct the trial and test spaces. We touch upon a moving least squares (MLS) approximants that are used in EFG method (Belytschko, Lu, and Gu [7], Dolbow and Belytschкo [8]) as well as in many of the other meshless methods (BeLYTschKo et. al [4]).

In the MLS approximation, the trial function $u^{h}$ is defined as:

$$
u_{L}^{h}(\mathrm{x}, \bar{x})=\sum_{j=0}^{k} p_{j}(x) a_{j}(\bar{x})=\mathbf{p}^{\mathbf{T}}(x) \mathbf{a}(\bar{x})
$$


where $\mathrm{p}(\mathrm{x})$ is a complete polynomial of order $k$. In order that the local approximation is the best approximation to $u(\mathrm{x})$ in a least-squares sense, the coefficient vector $\mathbf{a}(\bar{x})$ is selected to minimize the following least square discrete $L_{2}$ norm:

$$
\begin{gathered}
J(\mathbf{a}(\bar{x}))=\sum_{I} w\left(x-x_{I}\right)\left[u_{L}^{h}(\mathbf{x}, \bar{x})-u\left(x_{I}\right)\right]^{2} \\
=\sum_{I} w\left(x-x_{I}\right)\left[\mathbf{p}^{T}\left(x_{I}\right) \mathbf{a}(\bar{x})-u_{I}\right]^{2}
\end{gathered}
$$

where $w\left(\mathrm{x}-\mathrm{x}_{I}\right)$ is the weighting function. The functional $J_{\overline{\mathbf{x}}}(\mathbf{a})$ can be minimized by setting the derivative of $J_{\overline{\mathbf{x}}}(\mathbf{a})$ with respect to a equal to zero, hence $\partial J(\mathbf{a}) / \partial \mathbf{a}=0$.

In EFG method, each node is associated with a domain of influence $r$, which is the support of the isotropic weight function $w\left(\mathrm{x}-\mathrm{x}_{I}\right)$, with $w\left(\mathrm{x}-\mathrm{x}_{I}\right)>0$ in its interior and $w\left(\mathrm{x}-\mathrm{x}_{I}\right)=0$ outside it. Typically, domains of influence are circular or rectangular in 2D. Several weight functions can be used. A review of some of the possibilities can be found in (Belytschko et. all [4]). In this work, we use cubic spline weight functions with circular domain.

The approximation can be written shortly as:

$$
u^{h}(x)=\sum_{I=1}^{N} \phi_{I}(x) \hat{u}_{I}=<^{T}(x) \hat{\mathbf{u}}
$$

The EFG shape function $<(\mathrm{x})$ is defined as:

$$
<^{T}(x)=\mathbf{p}^{T}(x) \cdot[\mathbf{M}(x)]^{-1} \cdot \mathbf{B}(x)
$$

with

$$
\mathbf{B}(\mathrm{x})=\left[\begin{array}{llll}
w\left(x-x_{1}\right) \mathbf{p}\left(x_{1}\right) & w\left(x-x_{2}\right) \mathbf{p}\left(x_{2}\right) & \ldots & w\left(x-x_{N}\right) \mathbf{p}\left(x_{N}\right)
\end{array}\right]
$$

The continuity of the shape function is governed by the continuity of the basis function $\mathbf{p}(\mathrm{x})$.

\section{Discrete equations of THE GALERKin WEAK Form}

We consider the following two-dimensional problem, on the domain $\Omega$ bounded by $\Gamma$ :

$$
\Delta \sigma+\mathbf{b}=\mathbf{0} \text { in } \Omega
$$




$$
\begin{array}{cc}
\mathbf{n} \sigma=\overline{\mathbf{t}} & \text { on } \Gamma_{\mathrm{t}} \\
\mathbf{u}=\overline{\mathbf{u}} & \text { on } \Gamma_{\mathrm{u}}
\end{array}
$$

where $\sigma$ is the stress tensor, which corresponds to the displacement field $\mathrm{u}$ and $\mathrm{b}$ is a body force vector, $\bar{t}$ is the prescribed traction vector on Neumann boundary $\Gamma_{\mathrm{t}}, \bar{u}$ is the vector of prescribed displacements on Dirichlet boundary $\Gamma_{\mathrm{u}}, \Delta$ is a linear gradient operator and $\mathbf{n}$ is the matrix of direction cosine components of a unit normal to the domain boundary.

One of main characteristic of the meshless methods is that the shape functions do not satisfy the Kronecker delta condition at each node, i.e., $\varphi_{i}\left(x_{j}\right) \neq \delta_{i j}$.

So, the imposition of essential boundary conditions is more complicated than for the standard FEM. To enforce the essential boundary conditions, Lagrange multipliers are used in the potential energy functional.

Substituting the approximate solution $u^{h}(\mathrm{x})$ and the test function $\delta v$ constructed according to Eq. (3.1) into the variational principle using Lagrange multipliers, given by:

$$
\int_{\Omega} \varepsilon_{v}: \sigma d \Omega-\int_{\Omega} v u \mathbf{b} d \Omega-\int_{\Gamma_{t}} \delta \lambda v \overline{\mathbf{t}} d \Gamma-\int_{\Gamma_{u}} \delta \lambda(u-\overline{\mathbf{u}}) d \Gamma+\int_{\Gamma_{u}} \lambda v d \Gamma=0
$$

the following discrete equations of the weak form for regular EFG is obtained:

$$
\left[\begin{array}{cc}
\mathbf{K} & \mathbf{G} \\
\mathbf{G}^{T} & \mathbf{0}
\end{array}\right]\left\{\begin{array}{c}
\mathbf{u} \\
\lambda
\end{array}\right\}=\left\{\begin{array}{c}
\mathbf{f} \\
\mathbf{q}
\end{array}\right\}
$$

and:

$$
\begin{gathered}
\mathrm{K}_{\mathrm{ij}}=\int_{\Omega} \mathbf{B}_{i}^{T} \mathbf{D} \mathbf{B}_{j} d \Omega \\
f_{i}=\int_{\Gamma_{t}} \varphi_{i} \overline{\mathbf{t}} d \Gamma+\int_{\Omega} \varphi_{i} \mathbf{b} d \Omega \\
G_{i k}=-\int_{\Omega} \varphi_{i} \mathbf{N}_{\mathbf{k}} d \Gamma, \quad q_{k}=-\int_{\Omega} \mathbf{N}_{\mathbf{k}} \bar{u} d \Gamma
\end{gathered}
$$

where:

$$
\mathbf{B}_{i}=\left[\begin{array}{cc}
\varphi_{i, x} & 0 \\
0 & \varphi_{i, y} \\
\varphi_{i, y} & \varphi_{i, x}
\end{array}\right] \quad \mathbf{N}_{k}=\left[\begin{array}{cc}
N_{k} & 0 \\
0 & N_{k}
\end{array}\right]
$$


To integrate the terms $\mathrm{K}_{i j}$ in the discrete equations, it is necessary to use numerical quadrature since the integrals cannot be evaluated analytically. To overcome the difficulties and the errors due to Gauss quadrature, we employ the idea of quasi-Monte Carlo integration (Rosca and Leitão [11]), used also in the original formulation of the SPH.

\section{Numerical EXAMPLES}

For comparison of the integration purpose, we generalize the above formulation for the approximation of an arbitrary function. Let $\mathrm{u}(\mathrm{x})=1-\mathrm{x}^{2}$.

In this numerical example we consider isotropic weight function, where the support is circular. In this investigation, a cubic spline window function (Monaghan [10]) is used:

Using the approximation given by Eq. (2.1), the discrete form is obtained by numerical quadrature of the right hand side.

by Monte-Carlo Techniques:

$$
u^{h}(\mathrm{x})=\frac{1}{N} \sum_{I=1}^{N} w_{I} \cdot \hat{\mathbf{u}}_{I}
$$

or Gauss rule:

$$
u^{h}(\mathrm{x})=\sum_{I=1}^{N} w_{I}(\mathrm{x}) \cdot \Delta V_{I} \cdot \hat{\mathbf{u}}_{I}
$$

Various choices for the quadratures will be tested:

- random Monte-Carlo technique (as in original form of SPH, Sobol [9])

- quasi-random Monte-Carlo sequences (Sobol [9])

- Gaussian quadrature

For EFG formulations, the integration of the coefficients of the system equation given by Eq. (4.5) to Eq. (4.7) is performed using Gauss quadrature

Fig. 1 to Fig. 3 present comparison of the SPH solution to the exact solution for the displacements along the bar.

These results are obtained using eleven nodes equal distributed along the length of the bar. The integration was performed using the random Monte Carlo integration the quasi-random Monte-Carlo techniques and Gauss quadrature. For integration with Monte-Carlo techniques, a simple random generator was used; for Quasi-random Monte-Carlo integration, Weyl-sequences are adopted, while for Gauss integration, $4^{\text {th }}$ order quadrature rule are used.

Since Monte Carlo methods are statistical, for the integration with random Monte-Carlo, a high number of integration points are necessary. Even so, the results do not show a good accuracy. 
SPH and EFG approximation using MC integration

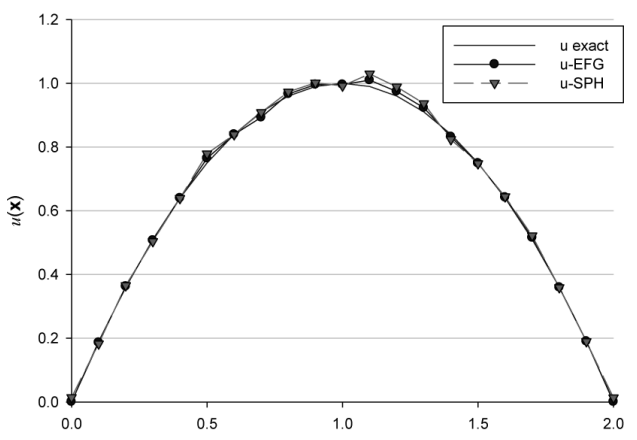

Fig. 1. SPH approximation of $u(\mathrm{x})=1-x^{2}$ with Monte Carlo approximation, for $r_{\mathbf{I}} / \mathrm{L}=0.02$.

SPH and EFG approximation using QMC integration

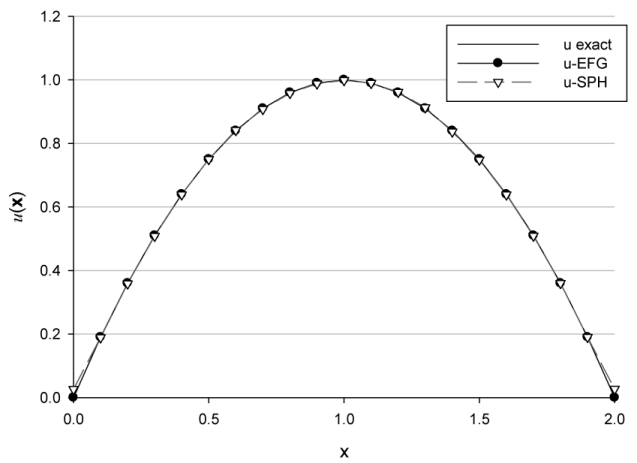

Fig. 2. SPH approximation of $u(x)=1-x^{2}$ with Quasi Monte Carlo approximation, for $r_{\mathbf{I}} / \mathrm{L}=0.02$.

SPH and EFG approximation using Gauss quadrature

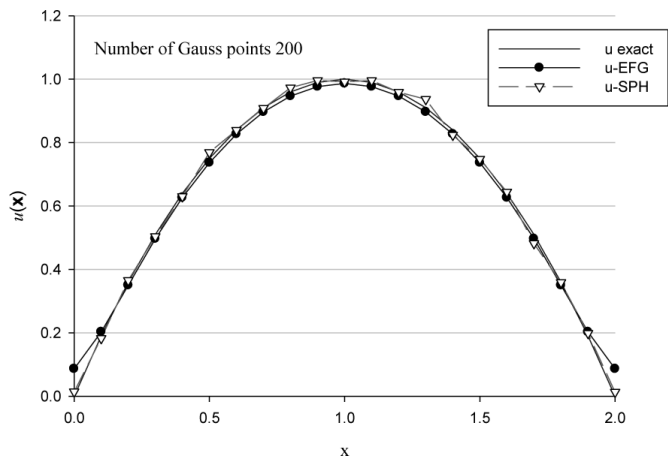

Fig. 3. SPH approximation of $u(x)=1-x^{2}$ with Gauss quadrature for $r_{\mathbf{I}} / \mathrm{L}=0.02$. 
The integration by quasi Monte Carlo techniques and Gauss quadrature give better results. We note that the numbers of integrations points used by Gauss and QMC techniques are comparable: for Gauss rule, a total of 200 integration points is enough, while for QMC to achieve the same accuracy, 500 integration points are needed.

In multidimensions, using Gauss quadrature, it is more difficult to come to grips with the need to increase the number of integration points, whereas for QMC the convergence rate, despite being always low, is independent of the dimension. This makes the use of QMC integration to be promising.

\section{Conclusions}

Although the two principles MLS and RKPM have their roots in very different areas, (the first has its origin in data fitting, the second in wavelet theory), the resulting interpolation functions are virtually the same ( $\mathrm{Li}$ and $\mathrm{Liu}$ [3]). The original $\mathrm{SPH}$ method usually provides not so accurate approximations when applied for solving PDEs with boundary conditions, and thus, it is necessary to improve the interpolation, or its derivatives, in some way.

The test has clearly demonstrated that the proposed Quasi Monte Carlo integration technique provides accurate results in and can be utilized successfully for these problems. The method may be extended for 3D meshless problems without too many problems.

\section{REFERENCES}

1. L.B. Lucy, A numerical approach to the testing of the fission thesis. The Astronomical Journal, 82, 12, 1013-1024, 1977.

2. W.K. Liu, S. Jun, Y.F. Zhang, Reproducing Kernel Particle Methods, International Journal for Numerical Methods in Fluids, 20, 1081-1106, 1995.

3. S. Li, W.K. Liu, Meshfree and particle methods and their applications. Applied Mechanics Review, 55, 1, 1-34, 2002.

4. T. Belytschio, Y. Krongauz, D. Organ, M. Fleming, P. Krysl, Meshless methods: An overview and recent developments, Computer Methods in Applied Mechanics and Engineering, 139, 3-47, 1996.

5. P.W. Randles, L.D. Libersky, Smoothed Particle Hydrodynamics: Some recent improvements and applications, Computer Methods in Applied Mechanics and Engineering, 139, 375-408, 1996.

6. P. Lancaster, K. Salkauskas, Surfaces generated by moving least squares methods, Mathematics of Computation, 37, 141-158, 1981.

7. T. Belytschкo, Y.Y. Lu, L. Gu, Element-Free Galerkin Methods, International Journal for Numerical Methods in Engineering, 37, 229-256, 1994.

8. J. Dolbow, T. Belytschкo, An Introduction to Programming the Meshless Element Free Galerkin Method, Archives of Computational Methods in Engineering, 5, 3, 207-241, 1998.

9. I.M. SовоL, The Monte Carlo Method, University of Chicago Press, 1974.

10. J.J. Monaghan, An introduction to SPH. Computer Physics Communications, 48, 89-96, 1988. 
11. V.E. Rosca, V.M.A. LeITÃo, Quasi-Monte Carlo mesh-free integration for meshless weak formulations. Engineering Analysis with Boundary Elements, 32, 6, 471-479, 2008.

\section{Extended abstract}

For solving a partial different equation by a numerical method, a possible alternative may be either to use a mesh method or a meshless method. A flexible computational procedure for solving 1D linear elastic beam problems is presented that currently uses two forms of approximation function (moving least squares and kernel approximation functions) and two types of formulations, namely the weak form and collocation technique, respectively. The approximations functions constructed in continuous or in discrete way are used as approximations of the strong forms of partial differential equations (PDEs), and those serving as approximations of the weak forms of PDEs to set up a linear system of equations to reproduce Element Free Galerkin (EFG) and Smooth Particle Hydrodynamics (SPH) meshless methods. To approximate the strong form of a PDE, the partial differential equation is usually discretized by specific collocation technique. The SPH is a representative method for the strong form collocation approach. To approximate the weak form of a PDE, Galerkin weak formulation is used.

Numerical applications for beam problems are obtained by implementing various quadrature techniques to perform the integrations of the system equations in EFG method or for discretize the continuous form of the displacement of the SPH. Numerical comparison between these two formulations performed in the aim to illustrate the difference between the formulations. 